\title{
Patient pathways and perceptions of hypertension treatment, management, and control in rural Bangladesh: a qualitative study
}

This article was published in the following Dove Press journal:

Patient Preference and Adherence

\author{
Aliya Naheed ${ }^{\prime}, *$ \\ Victoria Haldane $2, *$ \\ Tazeen H Jafar ${ }^{3,4}$ \\ Nantu Chakma' \\ Helena Legido-Quigley ${ }^{2}$ \\ On behalf of the COBRA- \\ BPS Study Group
}

\begin{abstract}
'Initiative for Noncommunicable Diseases, International Centre for Diarrhoeal Disease Research (icddr), Dhaka, Bangladesh; ${ }^{2}$ Saw Swee Hock School of Public Health, National University of Singapore, Singapore; ${ }^{3}$ Program for Health Services and Systems Research, Duke NUS Medical School, Singapore; ${ }^{4}$ Duke Global Health Institute, Duke University, Durham, NC, USA

*These authors contributed equally to this work
\end{abstract}

\begin{abstract}
Purpose: Hypertension is an increasing threat to global public health, a leading cause of premature death, and an important modifiable risk factor for cardiovascular and cerebrovascular disease. Despite evidence on the efficacy of antihypertensive medication for blood-pressure control and mortality prevention, a large proportion of individuals are undiagnosed and untreated, especially in resource-constrained settings. This qualitative study explored patient pathways to care, as well as knowledge of and adherence to hypertension care.
\end{abstract}

Methods: We conducted in-depth interviews with 20 hypertensive patients in two rural districts in Bangladesh. Interviews were conducted and transcribed in Bangla and translated to English. QSR NVivo 10 software was used for analyses. We mapped patient pathways and report here on patient experiences accessing care from local pharmacies, the government, and private clinics. Results: Overall, most patients reported hypertension awareness prior to diagnosis and were conscious about consequences of hypertension. However, patients had little knowledge about prevention and treatment strategies. Most patients considered hypertension an important disease, albeit reporting taking medication only when symptomatic. Patients were aware of dietary advice; however, they were largely sedentary. Qualified doctors in both private and government settings diagnosed hypertension in the majority of the patients, and some were diagnosed by an informal provider and a few reported self-care at home. Patients followed three pathways: specialized hospitals for acute care, private hospitals/local pharmacy for nonacute symptoms, and incidental hypertension identification while being treated for another condition.

Conclusion: We identify barriers to access to hypertension prevention and care that prevented patients from seeking and receiving treatment from government facilities. Challenges included a lack of support to enable community screening by government health workers, long waiting times, and inadequate supplies for hypertension treatment. Expanding community-health workers' scope in the dissemination of chronic-disease information may improve patient pathways to hypertension care in rural communities in Bangladesh.

Keywords: Bangladesh, hypertension, diagnosis, awareness, medication, barrier, qualitative

\section{Introduction}

Hypertension is an increasing threat to global public health: the global burden of hypertension is expected to increase from the current estimate of 1 billion affected individuals to 1.56 billion affected individuals by $2025 .{ }^{1}$ Hypertension is a leading cause of premature death and an important modifiable risk factor for cardiovascular and cerebrovascular disease. ${ }^{2}$ Hypertension is more prevalent in low- and middle-income countries, where the rate of occurrence is rising more quickly compared to high-income countries. ${ }^{3}$ Across South Asia, overall hypertension prevalence is estimated to be $27 \%{ }^{4}$
Correspondence: Tazeen H Jafar Program for Health Services and Systems Research, Duke NUS Medical School, 8 College Road, Singapore 169857

Tel +6566012582

Fax +65 65348632

Email tazeen.jafar@duke-nus.edu.sg 
There is robust evidence that blood-pressure-lowering medications are effective in reducing morbidity and mortality from hypertension and related cardiovascular diseases. ${ }^{5}$ However, a large proportion of individuals remain undiagnosed, untreated, and uncontrolled, especially in low- and middleincome countries. ${ }^{6}$ An added complexity is that despite its association with long-term adverse outcomes, hypertension is mostly asymptomatic and detected mainly via opportunistic screening. ${ }^{7}$ Indeed, evidence from the Prospective Urban Rural Epidemiology study has shown that across 17 countries, only $47 \%$ of those with hypertension were aware of it (43.6\% for low- and middle-income countries). ${ }^{8}$ Similarly, adherence among hypertensive patients is reportedly low, with some studies suggesting that up to $80 \%$ of hypertensive patients have low adherence to medication. ${ }^{9}$ Previous systematic reviews have identified barriers to hypertension control, including multiple challenges at the individual, service, and health-system levels. ${ }^{10,11}$ One systematic review of qualitative research explored lay perspectives on hypertension and drug adherence, and noted internal barriers, including adherence only while symptomatic and fear of side effects, as well as external factors, including finances, lack of time, and forgetfulness. ${ }^{12}$ However, the review comprised mostly studies from the USA and other high-income countries, and did not report on any studies from South Asia. Importantly, there is a need to address not only adherence but also upstream risk factors for hypertension and other chronic diseases. Projection models from Pakistan show that targeting major risk factors, such as diet, exercise, and tobacco use, could reduce the number of deaths from these diseases, with similar benefits predicted for similar South Asian countries. ${ }^{13,14}$

The COBRA-BPS study was piloted in Bangladesh, Pakistan, and Sri Lanka to assess long-term feasibility of public health interventions to lower blood pressure. ${ }^{15}$ The study reported promising results using a multicomponent intervention that included blood-pressure screening, home health education by community health workers (CHWs), training local providers, and providing checklists to document care. This manuscript seeks to provide complementary experiential evidence at the country level on patient knowledge of hypertension, as well as the ways in which patients in Bangladesh seek, obtain, and adhere to care for hypertension.

\section{Case-study setting: Bangladesh}

Bangladesh is a lower-middle-income country ${ }^{17}$ with a population of 160 million; $37 \%$ of the population are aged 30-70 years, with a life expectancy of 71 years for men and 73 years for women. ${ }^{18}$ It is estimated that noncommunicable diseases account for $59 \%$ of total deaths in Bangladesh. Of these deaths, $17 \%$ are attributable to cardiovascular disease. ${ }^{18}$

Overall prevalence of hypertension in Bangladesh is estimated to be $26.4 \%$, with higher prevalence in women (32.4\%) than men $(20.3 \%)$ and higher odds in those 60-69 years old than younger age-groups (adjusted odds ratio [AOR] 3.77, 95\% CI 3.01-4.72). ${ }^{23}$ Risk factors for hypertension in Bangladesh include high incidence of metabolic syndrome as well as lifestyle factors, such as obesity and diet with a high salt intake. ${ }^{19}$ We previously reported poor control of hypertension $(<20 \%)$ in rural areas of Bangladesh. ${ }^{15}$ Low adherence to antihypertensive medications has been implicated as a key contributing factor to poor blood-pressure control in Bangladesh, and is related to low socioeconomic status, disease knowledge, lack of an accompanying person to go with patients to health facilities, and inadequate information on hypertension from health-care providers. ${ }^{20}$

There are four key actors in the Bangladesh health system: government, private sector, nongovernmental organizations and donor agencies. ${ }^{21}$ The government is mandated to provide policy, regulation, and comprehensive health services. Government services include general health and family-planning services offered through district hospitals, subdistrict health complexes, union health and family-welfare centers and community clinics, and special care services offered through medical college hospitals and universities and specialized hospitals, generally known as tertiary facilities. The private sector consists of both the formal sector, comprising Western and traditional services offered at hospitals and clinics, and the informal sector, comprising providers of Western and traditional medicines, faith healers, and others without professional training. ${ }^{22}$ Either directly or through government partnerships, nongovernmental organizations and their donor agencies provide innovative health programs to lower socioeconomic groups with a particular focus on gender equity. ${ }^{23}$

\section{Methods}

\section{Sampling strategy}

This qualitative study is embedded within a larger trial conducted in Bangladesh in the districts of Tangail (population 3.2 million) and Munshiganj (population 1.4 million). The unit of randomization was a cluster defined by $250-300$ households defined by local administration according to $\mathrm{CHW}$ catchment area (each served by one to two CHWs). These clusters are grouped in geographically contiguous administrative units of 10-15 clusters in each unit (12 subdistricts in Tangail, six subdistricts in Munshiganj), such that each unit is served by one government clinic. First, ten administrative units were randomly selected, followed by a random selection 
of one cluster per unit, stratified by distance $(2 \mathrm{~km})$ from a government clinic, thereby ensuring adequate representation of clusters far from the clinic. Then, five clusters were randomized to the multicomponent intervention versus usual care, as explained in the COBRA-BPS protocol paper. ${ }^{24}$

This study was undertaken in four randomly selected clusters assigned to a multicomponent intervention in two districts in Bangladesh. The sampling strategy was purposive and included selecting respondents based on few characteristics as hypertensive status, whether controlled or uncontrolled (Table 1), sex, age-group, religion, and those who had already been recruited in the trial in randomly selected clusters. The rationale was to determine the preimplementation barriers and facilitators to hypertension care and use the same also to guide areas for improvement in strategies.

\section{Data collection}

The study involved in-depth interviews with 20 patients conducted by trained anthropologists of the International Centre for Diarrhoeal Disease Research (icddr), Bangladesh. Interviews were conducted in Bangla and audio-recorded following written consent. All interviews were transcribed in Bangla by the interviewers themselves and translated into English by a bilingual translator in the team. All translations were reviewed line by line by a bilingual anthropologist

Table I Patient characteristics

\begin{tabular}{|c|c|c|c|}
\hline & Female & Male & Total \\
\hline Sex & 9 & II & 20 \\
\hline \multicolumn{4}{|l|}{ Age-group, years } \\
\hline $40-49$ & 4 & 0 & 4 \\
\hline $50-59$ & 3 & 5 & 8 \\
\hline $60-69$ & 2 & 4 & 6 \\
\hline $70+$ & 0 & 2 & 2 \\
\hline \multicolumn{4}{|l|}{ Location } \\
\hline Lauhajang & 2 & 3 & 5 \\
\hline Mirzapur & 3 & 2 & 5 \\
\hline Sirajdikhan & I & 4 & 5 \\
\hline Tangibari & 3 & 2 & 5 \\
\hline \multicolumn{4}{|c|}{ Management of hypertension } \\
\hline Controlled (SBP/DBP & 5 & 3 & 8 \\
\hline \multicolumn{4}{|l|}{$<140 / 90 \mathrm{mmHg})$} \\
\hline Uncontrolled (SBP/ & 4 & 8 & 12 \\
\hline \multicolumn{4}{|l|}{$\mathrm{DBP} \geq \mathrm{I} 40 / 90 \mathrm{mmHg})$} \\
\hline \multicolumn{4}{|l|}{ Education } \\
\hline 0 years & 5 & 2 & 7 \\
\hline $\mathrm{I}-5$ years & I & 5 & 6 \\
\hline $6-10$ years & 2 & 4 & 6 \\
\hline $\mathrm{II}+$ years & I & 0 & I \\
\hline \multicolumn{4}{|l|}{ Religious group } \\
\hline Islam & 7 & 9 & 16 \\
\hline Hindu & 2 & 2 & 4 \\
\hline
\end{tabular}

Abbreviations: SBP, systolic blood pressure; DBP, diastolic blood pressure.
(NC) and double-checked by the principal investigator (AN). All interviewers were bilingual and trained in conducting the interviews using probes developed by HLQ, AN, and THJ in study-group meetings facilitated by HLQ, and detailed sessions at the icddr, Bangladesh by AN with support from NC. The training included a description of the research protocol, qualitative study method, and principles of ethical research.

Interviewers followed an interview guide (Table 2) for interview structure. The guideline was piloted with individuals with hypertension in a rural community outside the study setting, and necessary edits were done for customization for the local culture and norms. The guide covered such topic areas as hypertension knowledge, diagnosis, prevention, and treatment, access of services, receiving care, health-care experiences, and recommendations. These categories allowed for exploration of patient experiences, which enabled the development of patient pathways presented in this manuscipt.

\section{Ethical approval}

Ethical clearance was obtained by the ethical review committees at the icddr, Bangladesh, Duke National University of Singapore Medical School, Singapore, and London School of Hygiene and Tropical Medicine, London, UK. The study was registered with ClinicalTrials.gov (NCT02657746). Study interviewers read the informed consent in Bangla to all respondents, who were recruited following written informed voluntary consent in Bangla for participation and audio-recording. The research team securely stored all interview materials. The ethical review committee of the icddr, Bangladesh approved the consent procedures. We ensured participant confidentiality by quoting participants, using pseudonyms. Participants were offered the option to refuse any question and to withdraw from the study at any time without repercussion. The study team made efforts to ensure interviews took place in comfortable, private locations suitable to the respondent.

\section{Data analysis}

Two research team members (VH, HLQ) coded interviews using an inductive approach and thematic analysis. Coding was conducted using QSR NVivo 10 software and using techniques from grounded theory, including line-by-line analysis, use of the constant comparative method, and identification of deviant cases. ${ }^{25,26}$ Our approach used elements of Levesque's patient-centered access-to-care framework as an underpinning theory to understand how patients seek and access care. ${ }^{16}$ Participant recruitment ceased following thematic saturation. Interviews were recorded and transcribed in full. Each transcript contains the interview number, sex, condition, and age range of the participant. To maintain 
Table 2 Summary interview guide

\section{Knowledge and diagnosis}

- Did you experience any symptoms before you were diagnosed with hypertension?

- If yes, can you describe those symptoms of hypertension?

- Do you have other health problems?

- Can you tell me about your other health problems (the one that worries you the most)? (Ask about hypertension and diabetes)

- How did you decide to seek care? Did the family help in the process? Or any organization?

- Can we talk about your experience of this process of seeking care?

- To what extent do you think hypertension is an important disease?

- How much did you know about hypertension before your diagnosis? What were your information sources at the time and now?

Prevention and treatment

- Have you received any advice on preventive and control measures on hypertension from community health workers (CHWs)?

- Was the information received appropriate to your needs?

- Have you changed your behavior (diet and exercise) since knowing about the condition?

- What was the treatment that was first prescribed? Was it subsequently changed?

- Does a family member or organization help you with taking the medication?

- Did you have to pay anything out of pocket for the treatment or for traveling?

- Do you take other alternative medication?

- How were the health-care facilities you visited?

- What difficulties did you face during this process while seeking treatment?

- What in the process of treatment could have been handled better?

Access of services and receiving care

- Are there shortages of drugs and consumables? Or access problems to facilities? Discuss problems.

- Are there problems accessing home health education and other services from CHWs?

- Are there problems accessing subsidies or financial support for additional health services?

Health-care experiences and recommendations

- How would you assess your communication with health providers you have encountered?

- Do you have a trusting relationship with your GP?

- To what extent have you been kept informed about your treatment?

- How can home health education and other services provided by $\mathrm{CHWs}$ be improved?

- Have you heard of any initiative to improve prevention of hypertension?

- From your experience, what could be done to make life easier for people suffering from hypertension?

- Are there any changes that need to be made outside the health-care system?

Abbreviation: GP, general practitioner.

confidentiality, all names were reported as pseudonyms and all identifiers in data were removed.

\section{Results}

We report our findings in six main themes identified through analysis of patient responses. The first theme explores patients' awareness of hypertension as a disease, as well as knowledge and practices (both formal and informal) for its treatment and management. The second theme explores patients' reports of adherence to hypertension medications. The third theme describes the various pathways to care and continued management described by patients, beginning with their initial diagnosis and onward to maintenance of hypertension. The fourth theme discusses barriers to access hypertension care, including patient preferences, direct and indirect costs, and the role of local market pharmacies in mitigating unmet health-care needs posed by these barriers. The fifth theme explores patients' relationships with providers, emphasizing patients' expectations of care and perceived quality of care offered in the private, public, and informal sectors. The final theme discusses responses that report on unmet needs for access to health services, as well as expanded scope for CHWs in providing information and monitoring of noncommunicable diseases. Table 3 provides a summary of key themes and examples of evidence.

\section{Patients' awareness and knowledge of hypertension treatment, management, and control}

\section{Awareness of hypertension and consequences of hypertension}

Overall, most patients reported some awareness of hypertension prior to diagnosis, and the majority were conscious of the consequences of hypertension. As Adilah explained:

I heard from people [...] I heard about high blood pressure and I heard that patients may feel headache, but I didn't understand. Now I understand what is headache and what is high blood pressure. [IDI-BD-20, age 40-49 years, female] 
Table 3 Key themes and examples of evidence

\begin{tabular}{|c|c|}
\hline Key themes & Evidence \\
\hline $\begin{array}{l}\text { Patient awareness and knowledge } \\
\text { of hypertension treatment, } \\
\text { management, and control }\end{array}$ & $\begin{array}{l}\text { If I forget to take medicine, then I feel neck pain, headache, discomfort in head, then I try to drink lime } \\
\text { sherbet if lime is available at home [at] that time. After that, the blood pressure reduces a little, then I make } \\
\text { my sons buy me medicines. [IDI-BD-04, age 50-59 years, female] }\end{array}$ \\
\hline Adherence to medications & $\begin{array}{l}\text { I stopped taking medicine before this for } 3 \text { or } 4 \text { years. I felt better at that time. After that, I became sick again } \\
\text { and I am taking [medicine]. [IDI-BD-19, age } 40-49 \text { years, male] }\end{array}$ \\
\hline $\begin{array}{l}\text { Patient pathways to hypertension } \\
\text { diagnosis, treatment, and } \\
\text { management }\end{array}$ & $\begin{array}{l}\text { Yes, [a] few days ago, I bought medicine for blood pressure from the local pharmacy. Pharmacy man asked } \\
\text { what types of medicine. I said high blood-pressure medicine. I didn't measure my blood pressure, just took } \\
\text { the medicine. I felt better after taking the medicine. [IDI-BD-03, age 50-59 years, male] }\end{array}$ \\
\hline $\begin{array}{l}\text { Health-system barriers to } \\
\text { accessing hypertension care }\end{array}$ & $\begin{array}{l}\text { The government doctors neglect the patient ... they do not give any medicine. We need to go there and } \\
\text { stand, buy tickets, and many other hassles. That's why I do not go there. [IDI-BD-I3, age 50-59 years, female] }\end{array}$ \\
\hline $\begin{array}{l}\text { Health-provider relationships } \\
\text { with patients }\end{array}$ & $\begin{array}{l}\text { There is a dispensary at the market ... the person who gives medicine there is very close with me. He is very } \\
\text { good ... he sells medicine. He takes care of me more. [IDI-BD-I2, age } 70-79 \text { years, male] }\end{array}$ \\
\hline Ways forward and unmet needs & $\begin{array}{l}\text { It will be good. He [community health worker] can caution me. They could confirm if my current habits are } \\
\text { suitable for hypertension control ... I will be benefited of course. Hypertension will be reduced. [IDI-BD-I2, } \\
\text { age } 70-79 \text { years, male] }\end{array}$ \\
\hline
\end{tabular}

However, most reported low knowledge of strategies for prevention and treatment. For some patients, prior knowledge was linked to prior experience of hypertension among family members. After diagnosis, the majority of the patients reported a basic understanding of the symptoms typically associated with hypertensive urgency/emergency and consequences of hypertension, as well as the need for treatment, especially informal treatment for self-management of acute episodes, and lifestyle modifications. In an exceptional case, one patient framed hypertension as a complex disease that interacts with other comorbidities.

Patients described primarily interpersonal information sources on the health-care options available to them, including knowledge accrued through experience and from family, friends, and colleagues. Patients reported these sources providing information on lifestyle changes, medication adherence, and options for access to care. As Akash reported:

My neighbors and my friends also told [about hypertension]

when we met. They said if you have high blood pressure, just

take medicine; otherwise you may face problems. Many people

also discuss about it. [IDI-BD-05, age 50-59 years, male]

Patients' perceptions of hypertension were mixed. Some downplayed the personal significance of the condition, while recognizing it was serious. These patients reported taking medication as a mitigating factor in their view of hypertension, while some linked not worrying about hypertension as a component of self-management of their condition. The few patients who reported worrying about hypertension linked their worry to their knowledge of consequences of hypertension, in particular their fear of acute events. As Maryam explained:
I think it is a severe disease [...] I think it may increase anytime and tear off the neck veins. I may fall down anytime, I am afraid about this very much [...] It is important to me because I have experienced it and I realize it. I think my blood becomes warm and I think I will not survive. I mark it as a severe disease. That's why I take medicine regularly.

[IDI-BD-06, age 40-49 years, female]

There were limited reports on what patients believed to be the main risk factors of hypertension; however, one patient reported that labor and stress were important factors causing hypertension. Many patients considered hypertension an important disease. All patients reported on the consequences of high blood pressure, including heart attack, stroke, headache, and neck pain. Further, multiple patients reported the belief that high blood pressure could cause the neck vein to tear off.

\section{Patient knowledge and beliefs: treatment and management}

Patients framed blood-pressure maintenance and prevention of acute events as comprising blood-pressure monitoring and lifestyle modifications, as well as taking medication. Patients explained informal treatment of acute hypertension events. For some patients, acute episodes are informally self-managed through pouring water on the patient's head or eating sour things. One patient linked these treatments to lack of financial resources for medication. As Akash observed:

Many hypertensive patients are not able to receive treatment, due to their financial problem. Many of them only pour water on their head or eat something sour if their 
blood pressure increases suddenly. [IDI-BD-05, age 50-59 years, male]

Most patients reported awareness of the need to check their blood pressure as part of hypertension management. Patients who checked their blood pressure reported selfmotivation to monitor, including asking the doctor, as well as various other triggers that motivated them to monitor their blood pressure, including symptoms of hypertension, as well as locational cues, such as being at the market, where blood-pressure checks are available. While most patients reported monitoring blood pressure at the market pharmacy, either due to symptoms or because they were nearby, a few reported asking a private doctor to measure their blood pressure. One patient reported owning a blood-pressure monitor and taking measurements at home. Those who did not attend blood-pressure screenings reported that they did not screen because they felt well, or they only screened when they sought medical care for hypertension or otherwise, as well as such barriers as distance.

Patients described the need for multiple lifestyle adjustments to prevent and manage hypertension, including dietary adjustments, avoiding hard labor, taking up moderate exercise, and tobacco cessation. Generally, patients were given dietary advice, both from health-care providers and friends and family, and some reported being adherent to it; however, some reported nonadherence primarily for reasons of intolerance to taste, as well as fasting during Ramadan. Patients also differentiated between dietary advice for diabetic and hypertensive patients. As Hasan reported:

I don't eat too much beef and also don't take extra salt in meal. I will not lie about eating sweets. I eat sweets [...] I don't have diabetes, so I eat sweets as much as I can.

[IDI-BD-07, age 50-59 years, male]

Similarly, the majority of the patients reported receiving advice to take up moderate physical activity, such as walking, but adherence was variable, due to perception of exercise and barriers, such as the rainy season. A few patients reported that due to their condition, age, and at the behest of their families, they should rest and not do exercise. In an exception, one participant differentiated the activity needs of diabetic and hypertensive patients.

\section{Adherence to medication}

Patients reported knowing of the need to take medication and linked that directly to feeling better. Patients reported multiple medications available for hypertension treatment, including: Tenoloc, Camlotor, Amdocal 50 Plus, Angilock 50, Losartil 50, Tenoren, Tenocab, and Bisolol. While some patients did not know the names of the medication they were taking and identified it by color and shape, most knew the cost. A few patients reported on facilitators of adherence, primarily the role of family members in supporting adherence to medications. Patients spoke of children reminding them to take their medication, and one patient reported how his spouse put medication in his pockets to prevent him from missing a dose.

More widely reported were experiences of lapses in adherence resulting from feeling better. Most of the patients reported that they became nonadherent to prescribed medication after they felt better and only took medication when they became symptomatic. Some explained patchy adherence as a function of their belief that taking too much medicine for hypertension is not good. As Akash explained:

As I feel well now, so I don't take medicine. I can understand when my blood pressure becomes high. But I do not take always blood pressure medicine, because taking too much medicine for hypertension is not good. I am not taking medicine because I am feeling well for the last 6 months. [IDI-BD-05, age 50-59 years, male]

Asad, who runs a pharmacy, described the role of polypharmacy as a component of transient nonadherence:

I take many drugs like hypertension, pain, sleeping pill. Maybe I missed a dose the day before yesterday; that's why the pressure increased a little [...] maybe I forgot when taking the medicine one by one. I felt too hot at the evening. I checked my blood pressure and found it 140/110. After going to home, I took medicine at night and after taking the medicine it came under control. [IDI-BD-15, age 50-59 years, male]

Some patients described switching medications, a few reportedly changed either through visiting multiple doctors, or in some cases as directed by their primary doctor. As Maryam described:

It was tablet in yellow color. I have taken that medicine some days, and I feel sick again and visited the doctor again. The doctor measured my blood pressure and told, "This medicine can't control your blood pressure." Then he has prescribed a blue color medicine for me [...] after 5 months, the medicine was changed by the doctor and again I felt sick after 7-8 months later. [IDI-BD-06, age 40-49 years, female] 
Hasan described in detail his experience of trying many medications and how none of them gave him his desired results, leading him to not adhere to his prescribed medication and rather to adopt a lifestyle modification-based selfmanagement regime, which he reported as enabling him to maintain lowered blood pressure:

I am not doing treatment, because I am handling it [my hypertension] carefully. Only medicine can't cure the patients. If I take medicine and eat huge amount of beef with fat, I will not be cured. [IDI-BD-07, age 50-59 years, male]

\section{Patient pathways to hypertension diagnosis, treatment, and management}

Patients reported various pathways to diagnosis, treatment, and management of hypertension. Synthesis and analysis of patient pathways revealed multiple patient typologies. We trace the patient journey, beginning with the call to action that triggered their health-care seeking, followed by the decision of which facility to access, diagnosis, prescription, and finally where they accessed medicine and monitoring activities (Figure 1). Most patients reported few barriers to accessing screening, care, or medication offered through market pharmacies, whereas some barriers, mostly financial, were reported in accessing care through private clinics. Multiple barriers, including distance, financial, personal preference, and availability of medicines, diagnostics, and health-care providers, were reported in government settings.

\section{Patient pathway I}

In many patients, the pharmacy acts as an important access point for health services (Figure 2). Patients report easy access to pharmacies and trust of local providers, referring to them as "doctor" and receiving long-term chronic disease management and screening (Box 1). While these pharmacies are common and utilized, some participants described medication costs as a barrier to accessing medication.

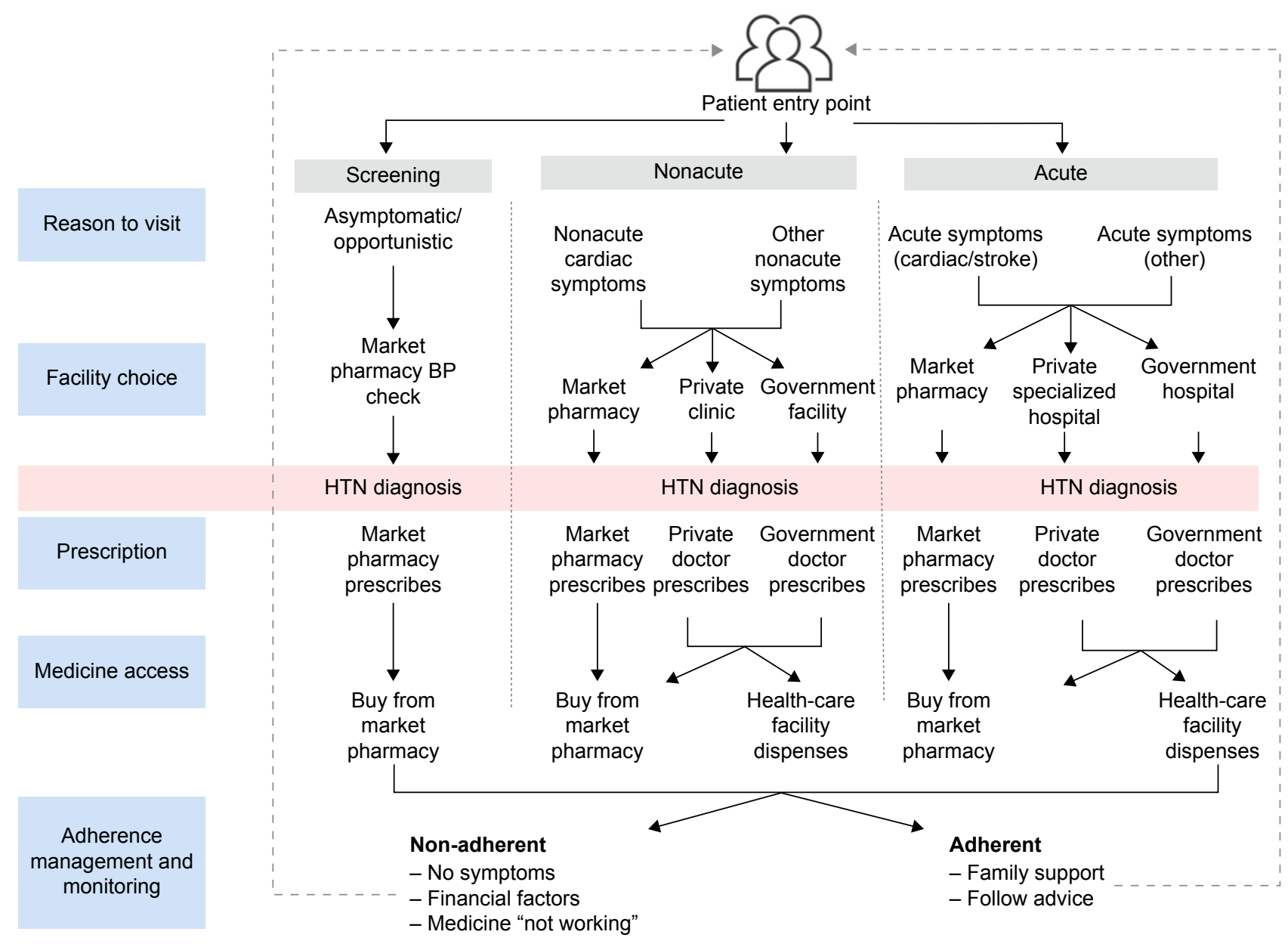

Figure I Overview of patient pathways.

Abbreviations: BP, blood pressure; HTN, hypertension. 


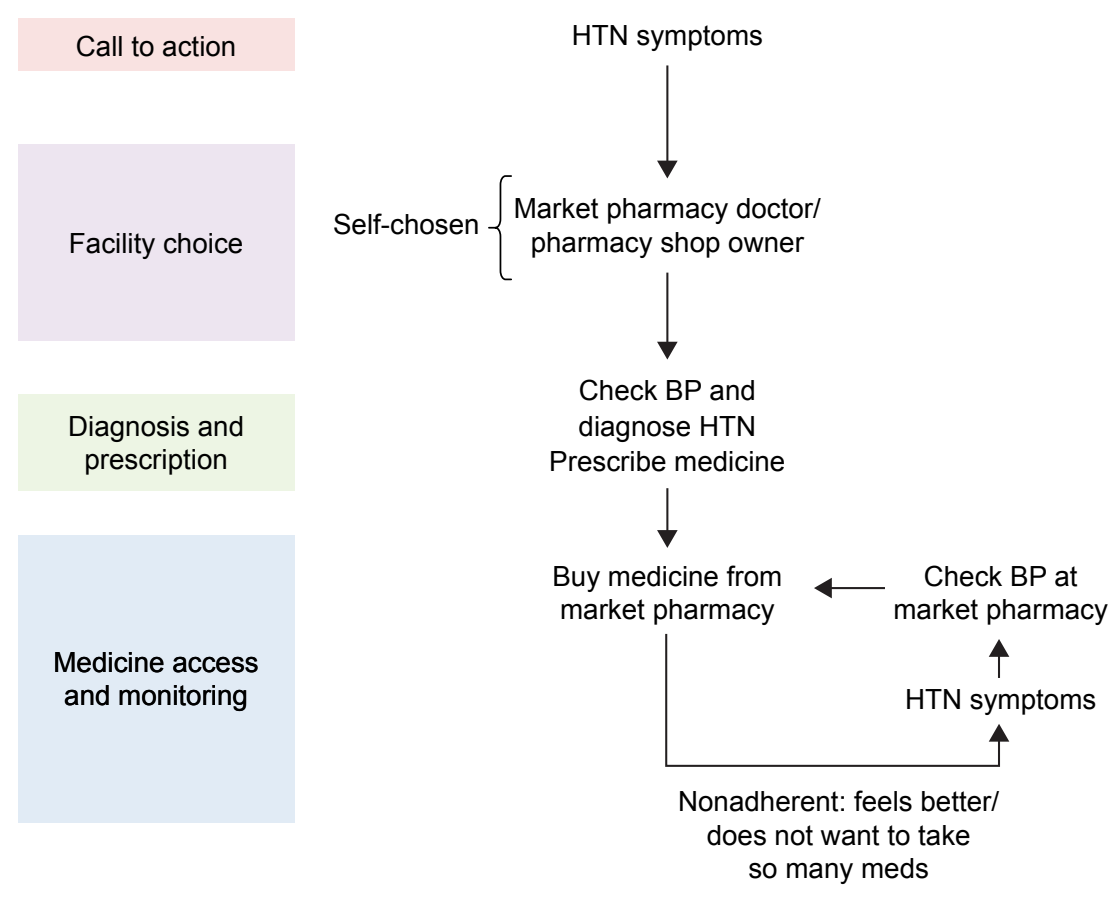

Figure 2 Patient pathway: nonacute symptoms.

Abbreviations: BP, blood pressure; HTN, hypertension.

\section{Patient pathway 2}

One typology describes an acute to a chronic trajectory, whereby an acute episode uncovers preexisting hypertension (Figure 3). In these episodes, families, friends, or those present during the episode often choose to convey the patient to care, thus playing a role in facility choice. Following this episode, patients enter the management phase, typified by accessing medicine at a local pharmacy and transiting to nonadherence, and upon reemergence of symptoms undergoing screening and reuptake of medication at the local pharmacy (Box 2). A small number patients will present to the health facility with manifestation of a hypertensive emergency or even malignant hypertension, or an acute cardiovascular event (heart attack or stroke) requiring immediate therapy.

\section{Patient pathway 3}

Patients also enter the health-care system for nonacute treatment of other conditions and are subsequently diagnosed

\section{Box I Example of a nonacute patient pathway}

Akash (IDI-BD-05, age 50-59 years, male) explained how he sometimes
checked his blood pressure in the pharmacy prior to his hypertension
diagnosis. He recalled that his blood pressure was increasing and
fluctuating. The pharmacy owner then provided him with medicine that
reduced his blood pressure. He took the medication from the pharmacist
until his blood pressure went into the normal range, and after achieving
normal blood pressure he stopped taking medicine, but continues to
sometimes check his blood pressure at the pharmacy and take medicine
when symptomatic.

with hypertension (Figure 4). Patients on complex pathways transit from government to pharmacy to private care, with choice influenced by perceived acuteness of symptoms or events (Box 3). Some patients were diagnosed with hypertension when they had long-term sequelae (such as heart failure) and presented to a pharmacy/clinic/hospital with subacute or chronic onset of symptoms associated with a complication or an unrelated illness. However, despite the common pathways for hypertension care, fewer patients sought care from government facilities for nonacute conditions, suggesting low preference for seeking care for hypertension from government facilities.

\section{Health-system barriers to accessing hypertension care}

Patients reported various health-system barriers impeding their ability to access hypertension treatment and management. In terms of health-care access within communities, a majority of the patients reported a lack of outreach into the community for chronic conditions, such as hypertension: participants reported that government outreach work addressed only vaccinations and family planning.

When accessing government health services and facilities, patients reported on long waiting times and lack of available doctors, diagnostics, and medicines. The informal sector was reported to be largely acceptable to patients as a means of treating and managing hypertension, with many 


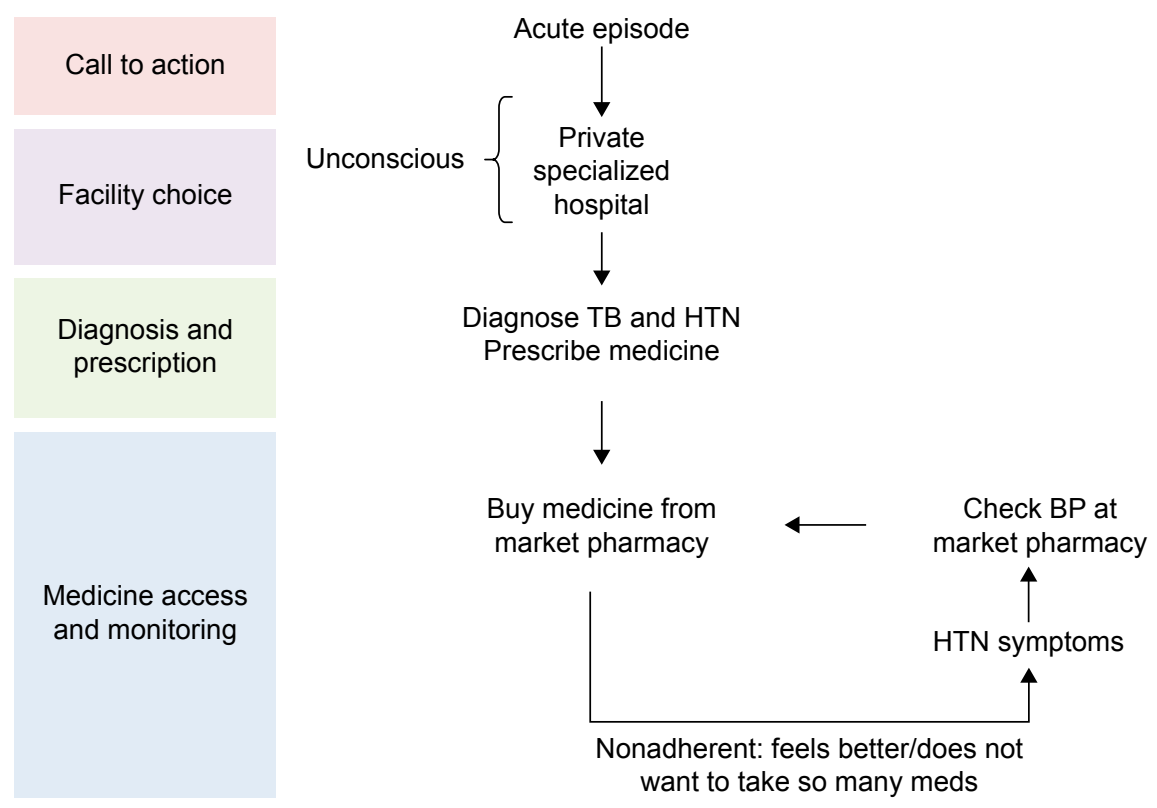

Figure 3 Patient pathway: acute episode.

Abbreviations: BP, blood pressure; HTN, hypertension; TB, tuberculosis.

reporting visiting local pharmacies for high-blood-pressure screening, as well as to purchase medications. As Zaytun explained:

The government doctors neglect the patient [...] they do not give any medicine. We need to go there and stand, buy tickets and many other hassles. That's why I do not go there. [IDI-BD-13, age 50-59 years, female]

Patients reported variably on the cost of care and medicine. Patients who took free medicine from the government clinics and those taking only medicines bought and prescribed at local pharmacies reported more favorably on cost. However, some patients reported that cost was a barrier to disclosure of illness, as well as adherence to medication. As Maymuna reported:

The cost is very high [...] suppose if I go to the [private] doctor and tell everything about all my problems, then

Box 2 Example of an acute patient pathway

Mohammed (IDI-BD-I4, age 60-69 years, male) described how he felt sick suddenly at work and was taken to a private specialized hospital unconscious. He recalled that prior to losing consciousness, his knee shook, and ascribes his feeling of weakness to too much stress and tension. However, while in hospital he was dually diagnosed with tuberculosis and hypertension. He took treatment for tuberculosis for I year and then began treating his hypertension. He explained how when his blood pressure increases, he feels dizzy or restless and numbness in his limbs; for him, a contributing perceived factor is lack of sleep. He does not take his medicines regularly, only when he feels symptoms of hypertension. the cost is like Tk800 [US\$9.55] or Tk1,000. So, I do not tell about all my problems. I just tell about the high blood pressure and take medicine. I just tell about the major problems, and then I take medicine for that $[\ldots]$ if you tell more problems, then the doctor will prescribe lots of medicines.

[IDI-BD-04, age 50-59 years, female]

Patients also reported on the cost of transport, time, and travel as a barrier to consistent formal care for hypertension. Some patients described the combination of these indirect costs and human-resource challenges in government clinics. As Hasan explained:

Sometimes I need to go there [government hospital], but I can't go, so I postpone to the day after. I go there the next day, but the doctor wasn't available. I wasted Tk20 on transport for no reason. [IDI-BD-18, age 60-69 years, male]

\section{Health-provider relationships with patients}

Many patients reported on the importance of the doctorpatient relationship and reported valuing doctors who spent time with them. However, some patients reported that especially in government hospitals, doctors did not have enough time to spend with them. Others reported how even when they saw the doctor, the experience did not meet their expectation. As Nusrat reported:

I needed more advice $[\ldots]$ such as we went to the doctor at the government hospital. He just listened to me and prescribed medicine but didn't ask anything about my problems. 


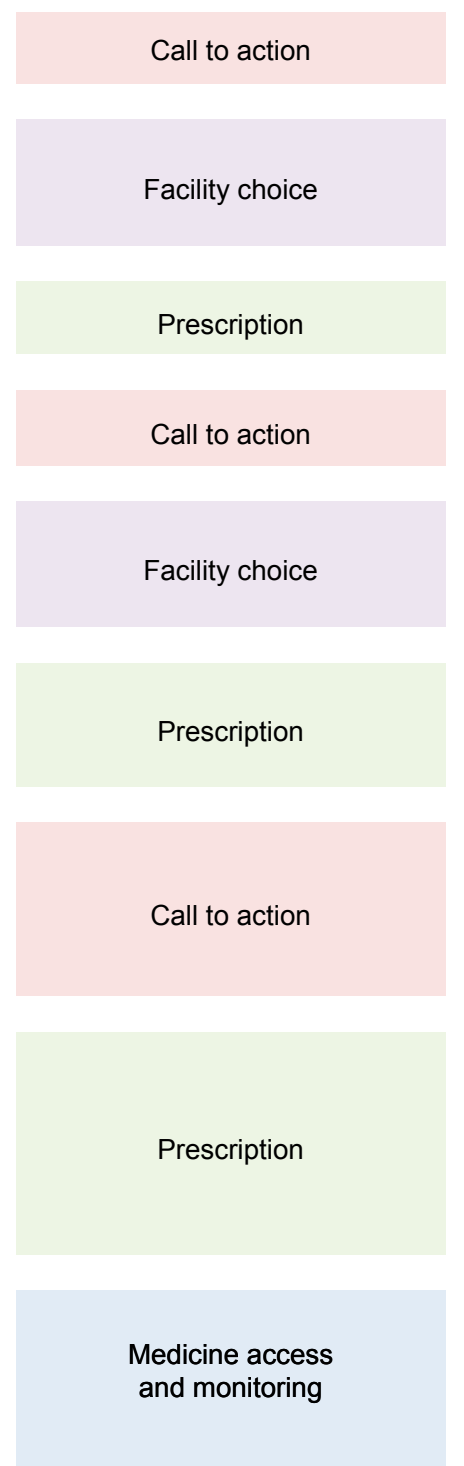

Figure 4 Patient pathway: comorbidity symptoms.

Abbreviations: HTN, hypertension; T2DM, type 2 diabetes mellitus.

Box 3 Example of an acute patient pathway

Asad (IDI-BD-15, age 50-59 years, male) felt neck pain, dizziness, and warmth in his body. He also felt dry throat and thirst that woke him up at night. He checked his blood pressure and blood sugar at the pharmacy, finding that both were high, and took medicine to control his conditions. Later, he experienced a stroke, and chose to go to a private hospital for treatment, where his hypertension was confirmed, and he was given different medicine for blood-pressure control. However, Asad does not feel like medications work for him, and after visiting many doctors, he has settled on a self-management regime based on medicine recommended to him via a text-message conversation he had with a friend who is a doctor. He now buys that from the market pharmacy and takes it to manage his hypertension.

He explains his belief that doctors and medicine alone cannot control hypertension and diabetes and that patients must eat right and self-manage.

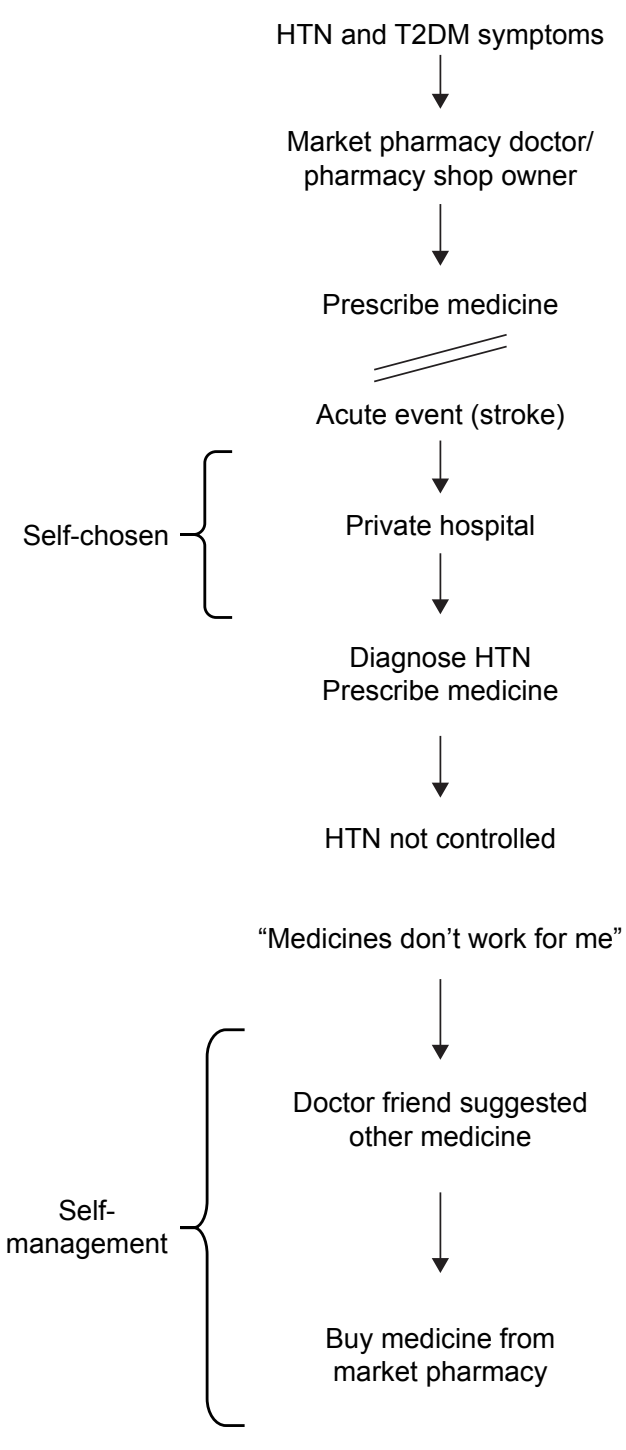

We hoped to get more information, but they weren't encouraging. [IDI-BD-19, age 40-49 years, female]

Among patients who reported preferring private care to public, the majority reported time spent with the doctor as a factor in their assessment that private care was of higher quality. Other patients, however, took a more transactional approach to receiving health care and did not report on the need for a relationship with their health-care provider. As Zayneb explained:

I went to him [the doctor], and I am now okay. I don't need to know more. I got my treatment, which I wanted - that's it. [IDI-BD-02, age 50-59 years, female] 
In the informal sector, patients reported on their relationships with local pharmacy providers. Patients described patronizing a specific pharmacy, and as Ali explained:

There is a dispensary at the market $[\ldots]$ the person who gives medicine there is very close with me. He is very good $[\ldots]$ he sells medicine. He takes care of me more. [IDI-BD-12, age 70-79 years, male]

\section{Ways forward and unmet need}

Patients identified multiple unmet needs and recommendations for better provision of hypertension treatment and management. By and large patients reported the need for access to doctors, medicine and diagnostics, especially in government health care settings. In terms of diagnostics, some patients reported the need for provision of affordable diagnostics to benefit patients. As Hasan explained:

Some tests like blood test, urine test should be available in the UHC [Upazila Health Complex], but here those tests are not available. Suppose it takes Tk100 for urine test at the outside, so if they took Tk30 or Tk50 for the same test at the UHC, then the public would be benefited. [IDI-BD-07, age $50-59$ years, male]

Patients also reported welcoming health-care outreach near their homes and the need for health education relating to noncommunicable diseases, such as hypertension. Many patients framed this type of outreach as needed to check that their habits are in line with medical advice. Asad reported:

I think it will be good [...] I will welcome it if I get advice that what should I do to control hypertension or become healthy [...] I will be benefited in terms of my physical condition. I can learn what I don't know. [IDI-BD-15, age 50-59 years, male]

Others reported that this type of outreach could provide an opportunity to link rural and poor individuals to care. As Mohammed explained:

It will be very good [...] such as there are many people at the rural areas still who don't dare to go to the doctors. Many people can't go for money. Many people don't know where to go. [IDI-BD-14, age 60-69 years, M]

\section{Discussion}

This qualitative study explored perceptions, knowledge, and experiences of patients accessing health care for hypertension and the pathways to seeking hypertension care in a populous low- and middle-income country rural setting. Patients reported knowledge of symptoms associated with hypertension; however, many reported knowing less about hypertension before they were diagnosed compared to after diagnosis. Most patients were aware of consequences of untreated hypertension. However, they reported several informal ways used to mitigate symptoms of hypertensive emergency, which is concerning and reflective of inadequate knowledge. Patients were diagnosed through a variety of means, such as hospitalization for acute conditions, visiting doctors for nonacute symptoms that may or may not have been related to complications of hypertension, and a few through screenings at market pharmacies. The role of pharmacies in diagnosis and prescribing of medication in low- and middle-income settings has been well documented, ${ }^{27,28}$ including in Bangladesh, where pharmacies are largely unregulated and mostly staffed by nonclinical salespeople trained through informal apprenticeships at these shops. ${ }^{29}$ In our study context, these pharmacies were utilized widely, especially for screening and medication purchases, including by patients who sought care in the formal sector. Patients reported that government hospitals would run out of medication, and thus even if patients were diagnosed and prescribed in a government-hospital setting, they would need to fill the prescription at a local pharmacy.

Clinical trial evidence has clearly demonstrated the benefit of lifestyle modification, including increase in physical activity and a diet rich in fruit and vegetables, low in salt, and low in saturated fat, in lowering blood pressure. ${ }^{30}$ We found that patients were generally aware of lifestyle modifications as a component of hypertension management. Patients reported knowledge of the need to reduce salt intake and avoid meat and dairy products. Many patients reported taking this advice once diagnosed with hypertension, even with small adjustments, such as eating less meat than before their diagnosis. In terms of physical activity, few patients undertook physical activity beyond household chores. Some patients reported that they should rest and not do physical activity. This is in line with other evidence, including a study of Bangladeshi patients living in the UK, who reported exercise as potentially exacerbating physical illness or weakness. ${ }^{31}$ Patients were also aware of the need to cease tobacco use; however, several participants reported still partaking in smokeless tobacco and betel on occasion and a few reported being unable to cease entirely. Other studies have reported on the link between betel, both with and without tobacco, and its contribution to high blood pressure, both globally ${ }^{32-35}$ and in Bangladesh. ${ }^{36}$ In terms of medication 
adherence, patients by and large reported that they would take medication to control symptoms of hypertension, but once they felt better would discontinue. These patients then reenter the health system to again access medication if they become symptomatic. This cycle between symptomatic medicationseeking and subsequent discontinuation has been reported extensively in other studies. ${ }^{2,37}$ Our findings underscore the importance of educating patients on the importance of adherence to prescribed antihypertensive therapy for lifelong maintenance of blood pressure at treatment targets for optimal prevention of cardiovascular and kidney disease.

Patients reported a variety of expectations and interactions with health-care providers. In the government-hospital setting, patients reported how doctors had to see many patients and thus had less time to devote to each patient. Some patients reported that this brevity was to be expected and felt they had received adequate care as long as they were prescribed medication. However, others felt they would have benefited from more time with the doctor at the government hospital, to receive more information on how to manage their condition. In the private sector, patients reported receiving adequate time and attention and described these as factors influencing their decision to seek private care. In the informal sector, some patients reported relationships with local pharmacists and trust in the information provided there. Other studies have described the importance of provider-patient relationships and communication in hypertension treatment and management, ${ }^{38}$ as well as in supporting adherence to treatment. ${ }^{39}$ In terms of this information gap, many patients reported that they would be welcoming of CHWs to visit their villages and homes. Patients reported that these workers would be beneficial in providing dietary and lifestyle advice and to provide a form of verification that their habits are appropriate.

\section{Strengths and limitations}

A strength of this manuscript is its exploration of patients' perspectives on the multiple health-system contact points they encounter, as well as the interaction between personal, social, and structural factors that enable or prevent them from accessing hypertension care. This was enabled by using Levesque's patient-centered access-to-care framework as a conceptual underpinning through which to view patients' perspectives. ${ }^{16}$ This study is one of the first to explore patients' experiences of hypertension in Bangladesh and capture their perspectives on treatment, management, and accessing the health system. The in-depth interviews allowed us to explore patient pathways through the health-care system and a diversity of experiences in seeking care for hypertension.
A limitation of the study is that of desirability bias, in that patients may positively frame and present their experiences. Also, as the sample was older, it is important to consider evidence that elderly patients tend to report higher levels of satisfaction than younger groups. ${ }^{40}$ Therefore, some of our positive reports on uptake of lifestyle advice and acceptance of health care may have been due to these effects. In addition, although we attempted to include a wide range of participants from various groups, including those in marginal groups or isolated, our sampling strategy is likely to have underrepresented those who are least connected to the health system.

\section{Conclusion}

This study reports on patient pathways to hypertension care in Bangladesh and patient perceptions on the treatment of hypertension, as well as the multiple barriers that prevent them from seeking and receiving care at government facilities. These patient perspectives are useful to consider in implementing programs or policies to address hypertension management and treatment in rural Bangladesh. Further, this study finds that expanding the scope for CHWs in the dissemination of the importance of diagnosis, treatment, and control of hypertension may improve patient utilization of primary-care facilities for the control of high blood pressure in rural communities in Bangladesh.

\section{Acknowledgments}

The authors would like to acknowledge all members of the COBRA-BPS Study group and the support teams and staff at the respective institutions for their contribution to the study. They would like to thank the data collectors for the efforts, and the study participants for their cooperation. COBRA-BPS is funded by the UK Department for International Development, the Medical Research Council, and the Wellcome Trust.

\section{Disclosure}

A Naheed is the country principal investigator from icddr, Bangladesh and TH Jafar is the overall chief principal investigator at Duke National University of Singapore Medical School, Singapore. The authors report no other conflicts of interest in this work.

\section{References}

1. Keamey P, Whelton M, Reynolds K, Muntner P, Welton P, He J. Global burden of hypertension: analysis of worldwide data. Lancet. 2005; 365(9455):217-223.

2. Legido-Quigley H, Lopez PC, Balabanova D, et al. Patients' knowledge, attitudes, behaviour and health care experiences on the prevention, detection, management and control of hypertension in Colombia: a qualitative study. PLoS One. 2015;10(4):e0122112. 
3. World Health Organization. Global Status Report on Noncommunicable Diseases. Geneva: WHO; 2014.

4. Neupane D, McLachlan C, Sharma R, et al. Prevalence of hypertension in member countries of South Asian Association for Regional Cooperation (SAARC): systematic review and meta-analysis. Medicine (Baltimore). 2014;93(13):e74.

5. Ettehad D, Emdin C, Kiran A, et al. Blood pressure lowering for prevention of cardiovascular disease and death: a systematic review and meta-analysis. Lancet. 2016;387(10022):957-967.

6. World Health Organization. A Global Brief on Hypertension: Silent Killer, Global Public Health Crisis. Geneva: WHO; 2013.

7. Patel K, Young L, Howell E, et al. Characteristics and outcomes of patients presenting with hypertensive urgency in the office setting. JAMA Intern Med. 2016;176(7):981-988.

8. Chow C, Teo K, Ranharajan S, et al. Prevalence, awareness, treatment, and control of hypertension in rural and urban communities in high-, middle-, and low-income countries. JAMA. 2013;310(9):959-968.

9. Elliott W. What factors contribute to the inadequate control of elevated blood pressure? J Clin Hypertens (Greenwich). 2008;10(1 Suppl 1):20-26.

10. Khatib R, Schwalm JD, Yusuf S, et al. Patient and healthcare provider barriers to hypertension awareness, treatment and follow up: a systematic review and meta-analysis of qualitative and quantitative studies. PLoS One. 2014;9(1):e84238.

11. Maimaris M, Paty J, Perel P, et al. The influence of health systems on hypertension awareness, treatment and control: a systematic literature review. PLoS Med. 2013;10(7):e1001490.

12. Marshall I, Wolfe C, McKevitt C. Lay perspectives on hypertension and drug adherence: systematic review of qualitative research. BMJ. 2012; $345: \mathrm{e} 3953$.

13. Jafar T, Haaland B, Rahman A, et al. Non-communicable diseases and injuries in Pakistan: strategic priorities. Lancet. 2013;381(9885): 2281-2290.

14. Misra A, Tandon N, Ebrahim S, et al. Diabetes, cardiovascular disease, and chronic kidney disease in South Asia: current status and future directions. BMJ. 2011;357:j1420.

15. Jafar T, de Silva H, Naheed A, et al. Control of blood pressure and risk attenuation: a public intervention in rural Bangladesh, Pakistan and Sri Lanka: feasibility trial results. J Hypertens. 2016;34(9):1872-1881.

16. Levesque J, Harris M, Russell G. Patient-centered access to health care conceptualizing access at the interface of health systems and populations. Int J Equity Health. 2013;12:18.

17. World Bank. World Bank country and lending groups. 2017. Available from: https://datahelpdesk.worldbank.org/knowledgebase/articles/ 906519-world-bank-country-and-lending-group. Accessed July 4, 2017.

18. World Health Organization. Noncommunicable diseases (NCD) country profiles: Bangladesh. 2014. Available from: http://www.who.int/nmh/ countries/bgd_en.pdf?ua=1. Accessed 4 July 2017.

19. Monwarul Islam A, Majumder A. Hypertension in Bangladesh: a review. Indian Heart J. 2012;64(3):319-323.

20. Hussain S, Boonshuyar C, Ekram A. Non-adherence to antihypertensive treatment in essential hypertensive patients in Rajshahi, Bangladesh. Anwer Khan Mod Med Coll J. 2011;2(1):9-14.

21. World Health Organization Regional Office for the Western Pacific. Bangladesh Health System Review. Manila: WPRO; 2015.
22. Mahmood S. Health systems in Bangladesh. Health Syst Policy Res. 2012;1(1):1-4.

23. Chowdhury A, Bhuiya A, Chowdhury M, Rasheed S, Hussain Z, Chen L. The Bangladesh paradox: exceptional health achievement despite economic poverty. Lancet. 2013;382(9906):1734-1745.

24. Jafar T, Jehan I, de Silva H, et al. Multicomponent intervention versus usual care for management of hypertension in rural Bangladesh, Pakistan and Sri Lanka: study protocol for a cluster randomized controlled trial. Trials. 2017;18(1):272.

25. Charmaz K. Constructing Grounded Theory: A Practical Guide Through Qualitative Analysis. London: Sage; 2006.

26. Strauss A. Qualitative Analysis for Social Scientists. Cambridge: Cambridge University Press; 1987.

27. Azhar S, Hassali M, Ibrahim M, Ahmad M, Masood I, Shafie A. The role of pharmacists in developing countries: the current scenario in Pakistan. Hum Resour Health. 2009; 7:54.

28. Miller R, Goodman C. Performance of retail pharmacies in low- and middle-income Asian settings: a systematic review. Health Policy Plan. 2016;31(7):940-953.

29. Ahmed S, Naher N, Hossain T, Rawal L. Exploring the status of retail private drug shops in Bangladesh and action points for developing an accredited drug shop model: a facility based cross-sectional study. J Pharm Policy Pract. 2017;10:21.

30. Sacks F, Svetkey L, Vollmer W, et al. Effects on blood pressure of reduced dietary sodium and the Dietary Approaches to Stop Hypertension (DASH) diet. $N$ Engl J Med. 2001;344(1):3-10.

31. Greenhalgh T, Helman C, Chowdhury A. Health beliefs and folk models of diabetes in British Bangladeshis: a qualitative study. BMJ. 1998;316(7136):978-983.

32. Guh J, Chen H, Tsai J, Chuang L. Betel-quid use is associated with heart disease in women. Am J Clin Nutr. 2007;85(5):1229-1235.

33. Tseng C. Betel nut chewing is associate with hypertension in Taiwanese type 2 diabetic patients. Hypertens Res. 2008;31(3):417-423.

34. Yen A, Chen L, Chiu Y, Boucher B, Chen T. A prospective communitypopulation-registry based cohort study of the association between betel-quid chewing and cardiovascular disease in men in Taiwan. Am J Clin Nutr. 2008;87(1):70-78.

35. Zhang L, Yang Y, Xu Z, Gui Q, Hu Q. Chewing substances with or without tobacco and risk of cardiovascular disease in Asia: a metaanalysis. J Zhejiang Univ Sci B. 2010;11(9):681-689.

36. Heck J, Marcotte E, Argos M, et al. Betel quid chewing in rural Bangladesh: prevalence, predictors and relationship to blood pressure. Int J Epidemiol. 2012;41(2):462-471.

37. Connell $\mathrm{P}, \mathrm{McKevitt} \mathrm{C}$, Wolfe $\mathrm{C}$. Strategies to manage hypertension: a qualitative study with black Caribbean patients. $\mathrm{Br} J$ Gen Pract. 2005;55(514):357-361.

38. Schoenthaler A, Chaplin W, Allegrante J, et al. Provider communication effects [sic] medication adherence in hypertensive African Americans. Patient Educ Couns. 2009;75(2):185-191.

39. Zolnierek KH, DiMatteo M. Physician communication and patient adherence to treatment: a meta analysis. Med Care. 2010;47(8):826-834.

40. Calnan M, Almond S, Smith N. Ageing and public satisfaction with the health service: an analysis of recent trends. Soc Sci Med. 2003;57(4): $757-762$.
Patient Preference and Adherence

\section{Publish your work in this journal}

Patient Preference and Adherence is an international, peer-reviewed, open access journal that focuses on the growing importance of patient preference and adherence throughout the therapeutic continuum. Patient satisfaction, acceptability, quality of life, compliance, persistence and their role in developing new therapeutic modalities and compounds to optimize

\section{Dovepress}

clinical outcomes for existing disease states are major areas of interest for the journal. This journal has been accepted for indexing on PubMed Central. The manuscript management system is completely online and includes a very quick and fair peer-review system, which is all easy to use. Visit http://www. dovepress.com/testimonials.php to read real quotes from published authors. 